\title{
ON A SUBRECURSIVE HIERARCHY AND PRIMITIVE RECURSIVE DEGREES $\left({ }^{1}\right)$
}

\author{
BY \\ PAUL AXT
}

1. Prior to the Herbrand-Gödel-Kleene definition of general recursive function, certain classes of functions defined more restrictively by particular "recursions" had been studied. Subsequently subclasses of the general recursive functions have continued to be of interest, not only because of the simplicity and naturalness of certain types of recursive definition, but because of the insight such classes might provide into recursiveness and effectiveness and because of the need for some measurement of the level or complexity of recursiveness of a function or predicate.

Pursuit of this interest led therefore to the devising of various hierarchies of recursive functions. It would seem that such a hierarchy ought to satisfy at least the following conditions: (1) that it be generated on the basis of some general principle, (2) that the order of a class should correspond to the complexity of the functions it contains in the sense that functions which enumerate or majorize a given class should appear in a higher class, (3) that the union of its classes should contain all the recursive functions or, if this fails, should be sufficiently large relative to one's purpose for the hierarchy.

The hierarchies of recursive functions which have been studied fall short of fully satisfying these conditions (cf. $[4, \S 1]$ ).

In [4] Kleene makes a new attempt at a classification of general recursive functions, by using the notion of relative primitive recursiveness and of the uniform effective enumerability of the functions primitive recursive in an assumed function. A general recursive function $h_{y}$, and a class $C_{y}$ of the functions primitive recursive in $h_{y}$, is associated with each element $y$ of a system $O$ of ordinal notations. If $y<_{o} z, h_{y}$ is primitive recursive in $h_{z}$ but $h_{z}$ is not primitive recursive in $h_{y}$, so $C_{y} \subset C_{z}$ and $C_{y} \neq C_{z}$. In addition the relation of primitive recursive equivalence is used to divide the number-theoretic functions into equivalence classes called primitive recursive degrees which may be studied after the manner of [5].

We shall investigate some problems connected with these classifications. First a uniqueness property of classes $C_{y}$ associated with notations for the same ordinal is described and shown to hold at ordinals less than $\omega^{2}$ and to fail at $\omega^{2}$. The $k$-recursive functions of Peter [6] are located in the hierarchy below the $\omega^{\omega}$ level. Although it is not yet settled whether all recursive functions are obtained, it is clear that $\bigcup_{y \in O} C_{y}$ is a large and interesting class.

Received by the editors December 9, 1957.

(1) A dissertation at the University of Wisconsin, written under the direction of Professor S. C. Kleene to whom the author expresses sincerest appreciation. 
Finally primitive recursive degrees are studied, and certain similarities to and differences from the theory of general recursive degrees of [5] are obtained.

2. For the hierarchy of classes $C_{y}$, the "uniqueness property" will be said to hold at an ordinal $\alpha$ if, whenever $y, z \in O,|y|=|z|=\alpha$, then $C_{y}=C_{z}$ (i.e. $h_{y}$ and $h_{z}$ are of the same primitive recursive degree). As is remarked in $[4, \S 7]$, the use of the $O$ of $S_{3}$, involving as it does general recursive fundamental sequences, would be out of keeping with the purpose of building a hierarchy based on primitive recursiveness. We now show that if the $O$ of $S_{3}$ is used, the uniqueness property fails at the first possible place in the hierarchy, namely at the $\omega$ level. In fact, the nonuniqueness occurs in such a way that a function of arbitrary primitive recursive degree for a general recursive predicate is definable at the $\omega$ level.

In this section $O$ refers to the $O$ of $S_{3}$.

If $\phi$ is a function in $C_{y}, y \in O$, we shall refer to an index of $\phi$ from $h_{y}$ $[4, \S 3]$ as a " $y$-index" of $\phi$. Let $e_{1}$ be an index of the primitive recursive function $\lambda b a\langle b, a\rangle$ (i.e. an index under $[4, \S 3]$ for $l=0$ ), and hence also a $y$-index of $\lambda b a\langle b, a\rangle$ for all $y \in O$. For $y, z \in O$, if $p$ is a $y$-index of $h_{2}{ }^{z}$, then

is a $y$-index of $h_{z}$.

$$
\left\langle 4,2, p,\langle 2,2,\langle 0,2,1\rangle\rangle, e_{1}\right\rangle
$$

Define the function $\zeta(n)$ primitive recursively as follows:

$$
\begin{aligned}
\zeta(0) & =\langle 0,2,1\rangle, \\
\zeta\left(n^{\prime}\right) & =\left\langle 4,2, \zeta(n),\langle 2,2,\langle 0,2,1\rangle\rangle, e_{1}\right\rangle .
\end{aligned}
$$

Now we can show by induction on $n$ that, for all $z \in O, \zeta(n)$ is a $\left(z+o n_{0}\right)$ index of $h_{z}$. In particular, taking $z=0_{o}, \zeta(n)$ is an $n_{O}$-index of $h_{0_{o}}(b, a)$, which is identically 0 . Thence it follows by induction on $n$, using the second recursion equation for $\zeta$ that, for $n \geqq m, \zeta(n)$ is an $m_{o}$-index of the constant function $\lambda b a$. Define the function fin $(y)$ by course-of-values recursion as follows:

$$
\text { fin }(y)=\left\{\begin{array}{l}
\text { fin }\left((y)_{0}\right)+1 \text { if } y=2^{(y)_{0}} \neq 1, \\
0 \text { otherwise. }
\end{array}\right.
$$

So if $y, z \in O, z=1$ or $z=3 \cdot 5^{(z)}$, and $y=z+o n_{o}$, then fin $(y)=n$.

Define $\eta(n)$ primitive recursively:

$$
\eta(n)=\langle 4,1, \zeta(n),\langle 2,1,\langle 2,1,1\rangle\rangle,\langle 3,1,1\rangle\rangle .
$$

The value $\eta(n)$ is a $y$-index of the one-place constant function 1 if $n<$ fin $(y)$, and (if $|y|<\omega$ ) of the constant function 0 otherwise.

If for each $n, u_{n}, v_{n} \in O$ and $v_{n}<_{o} v_{n+1}$, and $\lim _{n}\left|v_{n}\right|=\alpha$, we say the values of $u_{n}$ "run through" the values of $v_{n}$ if (1) for each $n$ there is an $m$ such that $u_{n}=v_{m}$, (2) for each $n u_{n}<_{o} u_{n+1}$, and (3) $\lim _{n}\left|u_{n}\right|=\alpha$.

Let $Q(n)$ be any recursive predicate. Define: 


$$
y_{n}=(2 n+1)_{o}
$$

and

$$
z_{n}= \begin{cases}y_{n}+o 1_{o} & \text { if } \bar{Q}(n) \\ y_{n} & \text { if } Q(n)\end{cases}
$$

Clearly the values of $y_{n}$ and of $z_{n}$ run through the finite notations, $n_{o}$. In addition $\lambda n y_{n}$ is primitive recursive and $\lambda n z_{n}$ is recursive. So for $u=3 \cdot 5^{y}$ and $v=3 \cdot 5^{z}$ where $\lambda n y_{n}=\lambda n\{y\}\left(n_{o}\right)$ and $\lambda n z_{n}=\lambda n\{z\}\left(n_{o}\right)$ (notation of $[1, \S 65]$ ), $h_{u}$ and $h_{v}$ are initial functions at the $\omega$ level. For every $n \geqq 0, \eta\left(\right.$ fin $\left.\left(y_{n}\right)\right)$ is a $z_{n}$-index $\left(\eta\left(\right.\right.$ fin $\left.\left(y_{n}\right) \div 1\right)$ is a $\left(z_{n}\right)_{0}$-index) of the constant function 1 if fin $\left(y_{n}\right)$ $<$ fin $\left(z_{n}\right)$ and of the constant function 0 otherwise. Therefore

$$
h_{z_{n}}\left(\eta\left(\text { fin }\left(y_{n}\right)-1\right), a\right)= \begin{cases}1 & \text { if fin }\left(y_{n}\right)<\text { fin }\left(z_{n}\right) \\ 0 & \text { otherwise }\end{cases}
$$

Furthermore $\left[\right.$ fin $\left(y_{n}\right)<$ fin $\left.\left(z_{n}\right)\right] \equiv\left[y_{n}<_{o} z_{n}\right] \equiv \bar{Q}(n)$. So $\lambda n h_{z_{n}}\left(\eta\left(\right.\right.$ fin $\left.\left.\left(y_{n}\right)-1\right), 1\right)$ is the representing function of $Q(n)$. And $\lambda n h_{z_{n}}\left(\eta\left(\right.\right.$ fin $\left.\left.\left(y_{n}\right)-1\right), 1\right)$ is primitive recursive in $\lambda n b a h_{z_{n}}(b, a)$. Therefore $Q(n)$ is primitive recursive in $\lambda n b a h_{z_{n}}(b, a)$, and hence in the initial function $h_{v}$. So if, in particular, a recursive $Q$ is chosen which is not primitive recursive in $h_{u}$, then $h_{v}$ is not primitive recursive in $h_{u}$, where $u$ and $v$ are the numbers defined above.

3. We now restrict ourselves to the system $O$ of ordinal notations (called $O^{\prime}$ in $\$ \$ 10$ and 11 of [4]) which differs from the $O$ of $S_{3}$ by the exclusive use of primitive recursive fundamental sequences of notations in the formation of limit notations and the use of indices instead of Gödel numbers. With this restriction on $O$ we prove the following theorem:

THEOREM 1. If $\alpha<\omega^{2}$, then the uniqueness property holds for $\alpha$.

Proof, by course-of-values induction up to $\omega^{2}$.

CAsE 1. $\alpha=\left|2^{y}\right|$. If $\alpha<\omega$, the uniqueness property holds because the ordinal notation is unique. Otherwise the argument of $[4, \S 6]$ applies.

CASE 2. $\alpha=\left|3 \cdot 5^{y}\right|$. By a "complete sequence" of ordinal notations between $l \cdot \omega$ and $(l+1) \cdot \omega$ is meant the sequence of values of a function $\lambda n z_{n}$ such that $z_{0} \in O,\left|z_{0}\right|=l \cdot \omega$, and $z_{n^{\prime}}=2^{z_{n}}$. If $3 \cdot 5^{z} \in O$ and $\left|3 \cdot 5^{z}\right|=(l+1) \cdot \omega$, then the values of the function $\lambda n z_{n}=\lambda n \operatorname{pr}\left(z,\left\langle n_{o}\right\rangle\right)$ must form either a complete sequence or (except possibly for a finite initial sequence of function values) a subsequence which runs through a complete sequence. Now let $v=3 \cdot 5^{z}$ and $u=3 \cdot 5^{y}$, where $y_{n}=\operatorname{pr}\left(y,\left\langle n_{O}\right\rangle\right)$ and $\alpha=|u|=|v|$. Then to prove Case 2 we shall compare $C_{u}$ and $C_{v}$, first where $y_{n}$ is such a subsequence of a complete sequence $z_{n}$, second where $y_{n}$ except for a finite initial sequence is such, and third where $y_{n}$ and $z_{n}$ are complete sequences.

To make the first of these comparisons, note that for all $n, z_{n} \leqq o y_{n}$ and 
$C_{z_{n}} \subset C_{y_{n}}$ (cf. [4 (13b)]). Recalling the properties of the functions $\zeta$ and fin of $\S 2$, observe that $\zeta\left(\right.$ fin $\left.\left(y_{n}\right)-n^{\prime}\right)$ is a $\left(y_{n}\right)_{0}$-index of $h_{z_{n}}$ provided fin $\left(y_{n}\right)>n$, and that $\lambda n \zeta\left(\right.$ fin $\left.\left(y_{n}\right)-n^{\prime}\right)$ is primitive recursive. Then we may write:

$$
h_{v}(b, a)=\left\{\begin{aligned}
h_{u}\left(\left\langle\zeta\left(\text { fin }\left(y_{(b)_{1}}\right)-(b)_{1}^{\prime}\right),(b)_{1}\right\rangle,\left\langle(b)_{0}, a\right\rangle\right) & \\
& \text { if } z_{(b)_{1}} \neq y_{(b)_{1}}, \\
h_{u}(b, a) & \text { if } z_{(b)_{1}}=y_{(b)_{1} .}
\end{aligned}\right.
$$

Thus $h_{v}$ is primitive recursive in $h_{u}$.

It remains to be shown that $h_{u}$ is primitive recursive in $h_{v}$. Denote $z_{y_{n}}$ by $r_{n}$ and $z_{y_{n}-1}$ by $s_{n}$. Since $n<z_{n}, y_{n}<{ }_{o} r_{n}$ and $C_{y_{n}} \subset C_{r_{n}}$. So $h_{y_{n}}$ is primitive recursive in $h_{s_{n}}$ with index $\zeta\left(y_{n}-\left(\text { fin }\left(y_{n}\right)\right)^{\prime}\right)$ and we can write

$$
h_{w_{n}}(b, a)=h_{r_{n}}\left(\zeta\left(y_{n}-\left(\text { fin }\left(y_{n}\right)\right)^{\prime}\right),\langle b, a\rangle\right) .
$$

Thus $\lambda n b a h_{y_{n}}(b, a)$ is primitive recursive in $\lambda n b a h_{r_{n}}(b, a)$. Further $\lambda n b a h_{r_{n}}(b, a)$ is primitive recursive in $\lambda n b a h_{z_{n}}(b, a)$ since $\lambda n y_{n}$ is primitive recursive. And hence $\lambda n b a h_{y_{n}}(b, a)$ is primitive recursive in $\lambda n b a h_{z_{n}}(b, a)$, so $h_{u}$ is primitive recursive in $h_{v}$. Therefore $h_{u}$ and $h_{v}$ are of the same primitive recursive degree.

In case $y_{i}<_{o} z_{0}, i=0, \cdots, M-1$, but $z_{0} \leqq o y_{M}$, let $x_{n}=y_{n+M}$. Then $\lambda n x_{n}$ is a primitive recursive function running through the values of $z_{n}$. Write $x_{n}=\operatorname{pr}\left(x,\left\langle n_{0}\right\rangle\right)$ and $w=3 \cdot 5^{x}$. By the argument just completed $h_{w}$ and $h_{r}$ are of the same (primitive recursive) degree. The following equations show that $h_{w}$ and $h_{u}$ are also of the same degree.

$$
\begin{aligned}
& h_{w}(b, a)=h_{u}\left(\left\langle(b)_{0},(b)_{1}+M\right\rangle, a\right), \\
& h_{u}(b, a)=\left\{\begin{array}{l}
h_{w}\left(\left\langle t_{i}, 1\right\rangle,\left\langle(b)_{0}, a\right\rangle\right) \quad \text { if }(b)_{1}=i \quad(i=0,1, \cdots, M-1), \\
h_{w}\left(\left\langle(b)_{0},(b)_{1}-M\right\rangle, a\right) \text { if }(b)_{1} \geqq M,
\end{array}\right.
\end{aligned}
$$

where $t_{i}, i=0, \cdots, M-1$, is an $\left(x_{1}\right)_{0}$-index of $h_{y_{i}}$.

Finally we compare classes $C_{u}$ for which the values of the function $\lambda n \operatorname{pr}\left((u)_{2},\left\langle n_{0}\right\rangle\right)$ form a complete sequence.

Denote by $z_{n}$ and $y_{n}$ two complete sequences of notations between $l \cdot \omega$ and $(l+1) \cdot \omega=\alpha$. For each $n \geqq 0,\left|z_{n}\right|=\left|y_{n}\right|$ and hence by the hypothesis of the induction $h_{z_{n}}$ and $h_{y_{n}}$ are of the same primitive recursive degree. Let $t$ be an index of the primitive recursive function $\operatorname{tr}^{2}(b, c)$ of $[4, \S 4]$. Then using $[4, \S 6]$, if $r$ is an index of $h_{z_{n}}$ from $h_{y_{n}}$,

$$
\langle 4,2,\langle 0,2,1\rangle,\langle 4,2, t,\langle 3,2,1\rangle,\langle 2,2, r\rangle\rangle,\langle 3,2,2\rangle\rangle
$$

will be an index of $h_{z_{n}}$, from $h_{y_{n}}$. Let $c$ be an index of $h_{z_{0}}$ from $h_{y_{0}}$. Define $\rho(n)$ primitive recursively as follows:

$$
\begin{aligned}
\rho(0) & =c, \\
\rho\left(n^{\prime}\right) & =\langle 4,2,\langle 0,2,1\rangle,\langle 4,2, t,\langle 3,2,1\rangle,\langle 2,2, \rho(n)\rangle\rangle,\langle 3,2,2\rangle\rangle .
\end{aligned}
$$


Now by induction on $n, \rho(n)$ is an index of $h_{z_{n}}$ from $h_{y_{n}}$. Thus $h_{z_{n}}(b, a)$ $=h_{y_{n+1}}(\rho(n),\langle b, a\rangle)$, so $h_{v}$ is primitive recursive in $h_{u}$. Similarly $h_{u}$ is primitive recursive in $h_{v}$.

4. The uniqueness property fails at $\omega^{2}$, however; there exist $u, v$ such that $u, v \in O,|u|=|v|=\omega^{2}$, and $C_{u} \neq C_{v}$. To show this we shall first establish two lemmas.

4.1. Suppose $\phi\left(x_{1}, \cdots, x_{n}\right)$ is primitive recursive in $\theta\left(z, y_{1}, \cdots, y_{m}\right)$ with index $e$. The index $e$ corresponds to a particular primitive recursive derivation of $\phi$ from $\theta$ (cf. [4, §3]). Carrying out the computation of the value $\phi\left((a)_{0}, \cdots,(a)_{n-1}\right)$ for a given $a$ by the method corresponding to the index $e$ will require function values $\theta\left(z, y_{1}, \cdots, y_{m}\right)$ for a specific finite set $S$ of $(m+1)$-tuples $z, y_{1}, \cdots, y_{m} . S$ depends on $e$ and $a$ and on the function $\theta$. For the following lemma we define $\delta_{e}(a)$ to be the largest value of $z$ which appears in the $(m+1)$-tuples of $S$, or 0 if $S$ is empty. (Kleene has examples with $\lambda a \delta_{e}(a)$ not primitive recursive.)

Lemma 1. If $\phi\left(x_{1}, \cdots, x_{n}\right)$ is primitive recursive in $\theta\left(z, y_{1}, \cdots, y_{m}\right)$ with index e, then $\lambda a \delta_{\theta}(a)$ is primitive recursive in $\theta\left(z, y_{1}, \cdots, y_{m}\right)$.

Proof, by a course-of-values induction on the index e. Suppose true for all indices less than $e$.

CASE 0. $(e)_{0}=0$.

$$
\phi \text { is } \theta . \quad \delta_{e}(a)=(a)_{0} .
$$

CAses $1,2,3 .(e)_{0}=1,2,3 . \phi$ is primitive recursive and its definition based on $e$ is independent of $\theta$. $\delta_{e}(a)=0$.

CASE $4 .(e)_{0}=4$.

$$
\phi\left(x_{1}, \cdots, x_{(e)_{1}}\right)=\psi\left(\chi_{1}\left(x_{1}, \cdots, x_{(e)_{1}}\right), \cdots, \chi_{k}\left(x_{1}, \cdots, x_{(e)_{1}}\right)\right),
$$

where $k=(e)_{2,1}$.

$$
\begin{array}{r}
\delta_{e}(a)=\max \left[\delta_{(e)_{2}}\left(\left\langle\chi_{1}\left((a)_{0}, \cdots,(a)_{(e)_{1}-1}\right), \cdots, \chi_{k}\left((a)_{0}, \cdots,(a)_{(e)_{1}-1}\right)\right\rangle\right),\right. \\
\left.\delta_{(e)_{3}}\left(\left\langle(a)_{0}, \cdots,(a)_{(e)_{1}-1}\right\rangle\right), \cdots, \delta_{(e)_{k+2}}\left(\left\langle(a)_{0}, \cdots,(a)_{(e)_{1}-1}\right\rangle\right)\right] .
\end{array}
$$

Since $\chi_{1}, \cdots, \chi_{k}$ are primitive recursive in $\theta$ by the hypothesis of the lemma, and $\delta_{i}(a)$, for indices $i<e$, are primitive recursive in $\theta$ by the hypothesis of induction, and max is a primitive recursive function, $\delta_{e}(a)$ is primitive recursive in $\theta$.

CASE 5. $(e)_{0}=5$.

$$
\begin{aligned}
\phi\left(0, x_{2}, \cdots, x_{n}\right) & =\psi\left(x_{2}, \cdots, x_{n}\right), \\
\phi\left(x_{1}^{\prime}, x_{2}, \cdots, x_{n}\right) & =\chi\left(x_{1}, \phi\left(x_{1}, \cdots, x_{n}\right), x_{2}, \cdots, x_{n}\right) .
\end{aligned}
$$

Define $\delta_{e}(a)$ by course-of-values recursion on $a$ as follows (noting that when $(a)_{0}>0$, then $\left.\left\langle(a)_{0}-1,(a)_{1}, \cdots,(a)_{(e)_{1}-1}\right\rangle<a\right)$ : 


$$
\begin{aligned}
\delta_{e}(a)=\left\{\begin{array}{r}
\delta_{(e)_{2}}\left(\left\langle(a)_{1}, \cdots,(a)_{(e)_{1}-1}\right\rangle\right) \text { if }(a)_{0}=0, \\
\max \left[\delta_{e}\left(\left\langle(a)_{0}-1,(a)_{1}, \cdots,(a)_{(e)_{1}-1}\right\rangle\right),\right.
\end{array}\right. \\
\delta_{(e)_{3}}\left(\left\langle(a)_{0}-1, \phi\left((a)-1,(a)_{1}, \cdots,(a)_{(e)_{1}-1}\right),\right.\right. \\
\left.\left.\left.(a)_{1}, \cdots,(a)_{(e)_{1}-1}\right\rangle\right)\right] \text { if }(a)_{0}>0 .
\end{aligned}
$$

The functions $\delta_{(e)_{\mathbf{3}}}(a)$ and $\delta_{(e)_{\mathbf{3}}}(a)$ are primitive recursive in $\theta$ by the hypothesis of induction, $\phi$ is primitive recursive in $\theta$, and $\max$ is primitive recursive. Hence $\delta_{e}(a)$ is primitive recursive in $\theta$. This completes the proof.

From the definition of $\delta_{e}(a)$ it follows that if an index $e$ defines $\phi_{1}\left(x_{1}, \cdots, x_{n}\right)$ from $\theta_{1}\left(z, y_{1}, \cdots, y_{m}\right)$ and $\phi_{2}\left(x_{1}, \cdots, x_{n}\right)$ from $\theta_{2}\left(z, y_{1}, \cdots\right.$, $\left.y_{m}\right)$ respectively, and given any $a$, if, for all $z<\delta_{e}(a)$ and all $y_{1}, \cdots, y_{m}$,

$$
\theta_{1}\left(z, y_{1}, \cdots, y_{m}\right)=\theta_{2}\left(z, y_{1}, \cdots, y_{m}\right) \text {, }
$$

then $\phi_{1}\left((a)_{0}, \cdots,(a)_{n-1}\right)=\phi_{2}\left((a)_{0}, \cdots,(a)_{n-1}\right)$.

Lemma 2. Given any general recursive function $\phi(a)$, there is a general recursive function $\nu(a)$ of the form $\mu y R(a, y), R$ primitive recursive, such that $\nu(a)$ $>\phi(a)$ for all $a$ and $\nu(a)$ is monotone increasing.

Proof. Let $e$ be a Gödel number of $\phi$. Now by the proof of the normal form theorem $[1, \S \S 56-58], T_{1}(e, a, x) \rightarrow U(x)<x$. Hence $\mu x T_{1}(e, a, x)>\phi(a)$. So we may take $\nu(a)=\mu y(i)_{i \leq a} T_{1}\left(e, i .(y)_{i}\right)$.

4.2. We define for arbitrary $v \in O$ a monotone increasing function $\xi_{v}(a) \in C_{v^{*}}$ (where $v^{*}=v+o 1_{o}$, notation of $[2$, footnote 23$]$ ) which majorizes the one-place functions in $C_{v}$ (and of course those in $C_{w}$ for all $w<\iota^{v}$ ):

$$
\xi_{v}(a)=\prod_{i \leqq a} p_{i}^{h_{i}^{*}(i,\langle a\rangle)+1}
$$

where $p_{i}$ is the $(i+1)$-st prime number. To see that this function has the desired majorizing property, let $\alpha(a)$ be an arbitrary one-place function in $C_{v}$ with $v$-index $e$. Then for each $a \geqq e$ the function value $\xi_{v}(a)$ has as a factor $p_{e}^{h_{v} *(e,\langle a\rangle)+1}=p_{e}^{\alpha(a)+1}$. Hence $\xi_{v}(a)>\alpha(a)$ for all $a \geqq e$.

4.3. The set of ordinal notations $<_{o} u$ for some $u \in O$, ordered by $<_{o}$, we call the "segment" of $O$ determined by $u$. Extending the definition of $\$ 2$, we say the values of $y_{n}$ "run through" the segment determined iv $u \in O$ if, for each $n, y_{n}<_{o} y_{n^{\prime}}<o u$ and $\lim _{n}\left|y_{n}\right|=|u|$.

Let $\lambda n y_{n}=\lambda n \operatorname{pr}\left(y,\left\langle n_{o}\right\rangle\right)$ be a primitive recursive function the values of which run through the segment determined by $u$ and have $u$ as limit notation $3 \cdot 5^{y}$. We shall define a monotone increasing function $\xi(a)$ which majorizes the one-place functions of $C_{w}$ for all $w<o u$, and which under the assumption that the uniqueness property holds at $|u|$ will be contained in $C_{u}$.

Applying 4.2, there is an index $d$ which, for all $v \in O$, defines $\lambda a \xi_{v}(a)$ from $h_{v^{*} ;}$ so $\xi_{v}(a)=h_{v^{* *}}(d,\langle a\rangle)$. Let 


$$
\xi(a)=\prod_{i \leq a} p_{i}^{h^{* * *} i(d,(a))+1}
$$

It is clear that $\xi(a)$ is primitive recursive in $\lambda n b a h_{y^{* *} n}(b, a)$. Supposing that the uniqueness property holds at $|u|, \lambda n b a h_{y^{* *} n_{n}}(b, a)$ is primitive recursive in $h_{u}$, since putting $y_{n}^{* *}=\operatorname{pr}\left(s,\left\langle n_{o}\right\rangle\right),|u|=\left|3 \cdot 5^{s}\right|$ and $h_{y * *_{n}}(b, a)$ $=h_{3.5^{\circ}}(\langle b, n\rangle, a)$. Thus (under the assumption of the uniqueness property at $|u|), \xi(a) \in C_{u}$.

Also $\xi(a)$ is monotone increasing. Furthermore $\xi(a)$ majorizes the oneplace functions in classes $C_{w}$, for $w<_{o} u$, in the following way. If $\alpha(a) \in C_{w}$, then (using $[4,(13)]) \alpha(a)$ must appear in $C_{y_{n}}$ for some value of $n$; let its $y_{n}$-index be $e$. Then for all $a \geqq n$, the function value $\xi(a)$ has as a factor

$$
p_{n}^{h_{n}{ }^{* *}(d,\langle a\rangle)+1}=p_{n}^{\xi_{n}(a)+1} ;
$$

and for all $a \geqq e, \xi_{y_{n}}(a)>\alpha(a)$. Hence for all $a \geqq \max (e, n), \xi(a)>\alpha(a)$.

\subsection{Theorem 2. The uniqueness property does not hold at $\omega^{2}$.}

Proof. We shall contradict the assumption that the uniqueness property holds at $\omega^{2}$ by constructing two primitive recursive sequences $y_{n}$ and $z_{n}$ $\left(y_{n}=\operatorname{pr}\left(y,\left\langle n_{o}\right\rangle\right), z_{n}=\operatorname{pr}\left(z,\left\langle n_{o}\right\rangle\right), u=3 \cdot 5^{\nu}, v=3 \cdot 5^{z}\right)$ such that each sequence runs through a segment of the notations of $O$ for the ordinals less than $\omega^{2}$, but $z_{n}$ advances through the segment so slowly in comparison with $y_{n}$ that $C_{u}$ can be shown (under the assumption that the uniqueness property holds) to contain functions not in $C_{v}$.

First, consider some $w \in O,|w|=\omega^{2}$. Then by 4.2 with $v=w, \xi_{w}(a)$ majorizes all the one-place functions of $C_{w}$. So by the assumption that the uniqueness property holds at $\omega^{2}, \xi_{w}$ majorizes all the one-place functions of $C_{u}$ and of $C_{v}$. By Lemma 2 with $[4, \S 9]$ there is a primitive recursive predicate $R(a, y)$ such that $\nu(a)=\mu y R(a, y)$ is monotone increasing and for all $a, \nu(a)$ $>\xi_{w}(a)$. Thus $\nu(a)$ majorizes the functions of $C_{u}$ and $C_{v}$.

Now use the predicate $R$ just described to define a function $\psi$ primitive recursively as follows:

$$
\begin{aligned}
\psi(b, k, n) & =n \text { if } k=0, \\
& =\psi(b, k-1, n) \text { if } k>0 \&(i)_{i \leq \operatorname{fin}(n)} \bar{R}(k-1, i), \\
& =3 \cdot 5^{\mathrm{sb}^{1}{ }_{1}(b, k-1)}+o\left(\text { fin }(n)-\mu i_{i \leq \operatorname{fin}(n)} R(k-1, i)\right)_{o} \text { otherwise, }
\end{aligned}
$$

where $s b_{1}^{1}$ is the function defined in $[4, \S 10]$. By the recursion theorem for primitive recursive functions $[4, \S 10]$ there is a number $f$ which is an index of $\psi(k, n)=\psi(f, k, n)$. Let $\zeta(k, n)=\psi\left(k, n_{o}\right)$.

Observe that, as we can prove by induction on $k$ (using the monotonicity property of $\nu(a))$, the first $\nu(k)$ values $\zeta(k, 0), \cdots, \zeta(k, \nu(k)-1)$ of the function $\lambda n \zeta(k, n)$ are 
$0_{o}, 1_{o}, \cdots,(\nu(0)-1)_{o}$

$\zeta(1, \nu(0)), \zeta(1, \nu(0))+o 1_{o}, \cdots, \zeta(1, \nu(0))+o(\nu(1)-\nu(0)-1) o$,

$\zeta(k, \nu(k-1)), \zeta(k, \nu(k-1))+o 1_{o}, \cdots, \zeta(k, \nu(k-1))+o(\nu(k)-\nu(k-1)-1)_{o}$,

and the subsequent values of $\zeta(k, n)$ are obtained by successive additions $(+o)$ of $1_{o}$, where, for $l=1, \cdots, k, \zeta(l, \nu(l-1))=3 \cdot 5^{\mathrm{sb}}{ }_{1}(l, l-1)$ is a notation for $l \cdot \omega$ given by the sequence of values of the function $\zeta(l-1, n)$. Thus for $l=0, \cdots, k-1, \zeta(k, \nu(l))=3 \cdot 5^{\mathrm{sb}{ }_{1}(\gamma, l)}$ and $|\zeta(k, \nu(l))|=(l+1) \cdot \omega$.

Let $z_{n}=\zeta(n, n)$. Clearly $z_{n} \in O$. For each $n, \zeta(k, n) \leqq o \zeta(k+1, n)$; and for each $k, \zeta(k, n)<_{o} \zeta(k, n+1)$. Hence $z_{n}<_{o} z_{n+1}$. Furthermore for every $n$ and $l$, if $n \geqq \nu(l)$ then $\left|z_{n}\right| \geqq(l+1) \cdot \omega$. Hence $\lim _{n}\left|z_{n}\right|=\omega^{2}$. Thus the values of the primitive recursive function $\lambda n z_{n}$ run through a segment of notations for ordinals less than $\omega^{2}$.

Define $y_{n}$ as follows:

$$
y_{n}=\left\{\begin{array}{l}
0_{O} \text { if } n=0, \\
3 \cdot 5^{\mathrm{sb}^{\mathbf{1}_{1}(f, n-1)}} \text { otherwise. }
\end{array}\right.
$$

Then $\lambda n y_{n}$ is also primitive recursive, and $y_{n}$ and $z_{n}$ run through the same segment of notations for ordinals less than $\omega^{2}$. By induction on $n$, using the properties of $\nu(n)$ and $\zeta(k, n), z_{n}<_{o} y_{n}$ for every $n>0$.

We now show that $C_{u}$ and $C_{v}$ are not identical by observing that the function $\xi(a)$, defined as in 4.3 using the sequence of notations $y_{n}$ just defined (and by 4.3 , under the assumption that the uniqueness property holds at $\omega^{2}$, a function of class $C_{u}$ ), majorizes all the one-place functions of $C_{v}$.

Let $\alpha(a)$ be an arbitrary one-place function in $C_{v}$, and let $e$ be an index of $\alpha$ from $\lambda n b a h_{z_{n}}(b, a)$. Then $\delta_{e}(\langle a\rangle)$ (as defined in 4.1) is the largest value of $n$, for which values of the function $\lambda n b a h_{z_{n}}(b, a)$ are used in the computation based on $e$ of the function value $\alpha(a)$. By Lemma 1, $\lambda a \delta_{e}(\langle a\rangle)$ is primitive recursive in $\lambda n b a h_{z_{n}}(b, a)$. Hence the $\nu(a)$ we have defined majorizes $\delta_{e}(\langle a+1\rangle)$. Let $d_{1}$ be an index of $\lambda n b a \theta(\langle b, n\rangle, a)$ from $\theta$, and let $c$ be an arbitrary number such that

$$
c>\max \left(\mu z_{z>0}(t)_{t 3 z}\left(\nu(t-1)>\delta_{e}(\langle t\rangle)\right), \operatorname{tr}^{3}\left(e, d_{1}\right)\right) .
$$

Denote by $\lambda a \alpha_{c}(a)$ the function primitive recursive in

$$
\lambda n b a h_{\zeta(c-1, n)}(b, a)\left(=\lambda n b a h_{y_{c}}(\langle b, n\rangle, a)\right)
$$

with index $e$. Then $\alpha_{c}$ is primitive recursive in $h_{y_{c}}$ with index $\operatorname{tr}^{3}\left(e, d_{1}\right)$.

We shall now show that $\alpha_{c}(c)=\alpha(c)$. First note that the computation based on $e$ of $\alpha(c)$ requires values of $\lambda n b a h_{z_{n}}(b, a)$ only for values of $n \leqq \delta_{e}(\langle c\rangle)$ $<\nu(c-1)$. It follows from the properties of $\zeta(k, n)$ that $(t)(i)_{i<\nu(t)}\left[\zeta(t, i)=z_{i}\right]$ 
and hence $(i)_{i<p(c-1)}\left[h_{\zeta(c-1, i)}(b, a)=h_{z_{i}}(b, a)\right]$. Thus by the remark at the conclusion of Lemma $1, \alpha_{c}(c)=\alpha(c)$.

Furthermore $\alpha_{c} \in C_{y_{c}}$. Now by $4.3, \xi(a)$ majorizes the functions of $C_{y_{o}}$ in such a way that $(x)_{x \geq \max \left(\operatorname{tr}^{8}\left(e, v d_{1}\right), c\right)}\left[\xi(x)>\alpha_{c}(x)\right]$. For all $c$ under consideration, $c>\operatorname{tr}^{3}\left(e, d_{1}\right)$; hence $\xi(c)>\alpha_{c}(c)=\alpha(c)$. Thus $\xi$ majorizes $\alpha$. Now $\xi$ is independent of the choice of $\alpha$ and $\alpha$ is an arbitrary one-place function in $C_{v}$, so $\xi$ majorizes all one-place functions of $C_{v}$.

$\S 5$. We next investigate the problem of locating Péter's $k$-recursive functions $[6, \S 10]$ in the hierarchy of $[4]$.

5.1. First we extend the indexing of functions primitive recursive in assumed functions $[4, \S 3]$ to functions $k$-recursive in assumed functions for $k>1$.

A function $\phi$ is said to be " $k$-recursive" in a fixed list of assumed functions $\theta_{1}\left(a_{1}, \cdots, a_{m_{1}}\right), \cdots, \theta_{l}\left(a_{1}, \cdots, a_{m_{l}}\right)$ if it is introduced by one of the schemata ()$, \cdots$, (IV) of $[4, \S 3]$, or one of the following schemata $\left(\mathrm{V}_{j}\right)$, $j=1, \cdots, k$, where $n \geqq 0$ and $\psi_{1}, \cdots, \psi_{j}, \chi, \gamma_{1}^{(1)}, \cdots, \gamma_{j-1}^{(1)}, \gamma_{1}^{(2)}, \cdots$, $\gamma_{j-2}^{(2)}, \cdots, \gamma_{1}^{(j-2)}, \gamma_{2}^{(j-2)}, \gamma_{1}^{(j-1)}$ are functions previously introduced by applications of the schemata:

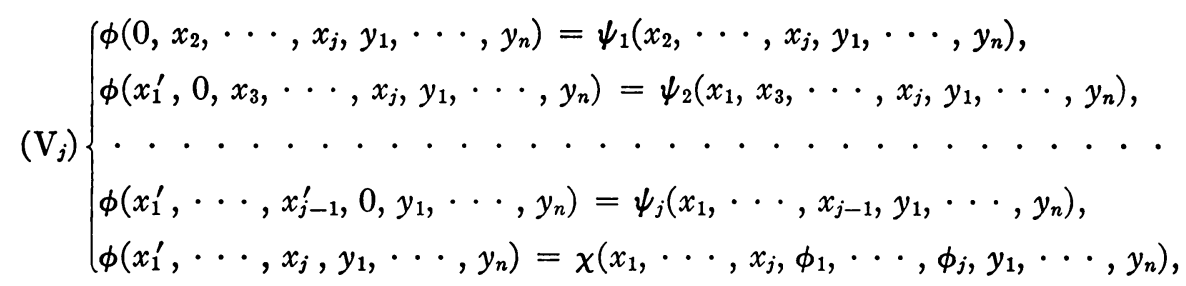

where

$$
\begin{aligned}
\phi_{i}= & \phi\left(x_{1}^{\prime}, \cdots, x_{i-1}^{\prime}, x_{i},\right. \\
& \gamma_{1}^{(i)}\left(x_{1}, \cdots, x_{j}, \phi\left(x_{1}^{\prime}, \cdots, x_{-1}^{\prime}, x_{j}, y_{1}, \cdots, y_{n}\right), y_{1}, \cdots, y_{n}\right), \cdots, \\
& \left.\gamma_{j-i}^{(i)}\left(x_{1}, \cdots, x_{j}, \phi\left(x_{1}^{\prime}, \cdots, x_{j-1}^{\prime}, x_{j}, y_{1}, \cdots, y_{n}\right), y_{1}, \cdots, y_{n}\right), y_{1}, \cdots, y_{n}\right) .
\end{aligned}
$$

For a given $k,\left(\mathrm{~V}_{k}\right)$ is the schema of $\left[6\right.$, middle p. 77] (but read " $\phi\left(n_{1}+1, \cdots\right.$, $\left.n_{k}+1\right)$ " for " $\phi\left(n_{1}, \cdots, n_{k+1}\right)$ "), except without the normalization to 0 of the value of $n_{1} \cdot n_{2} \cdots \cdots n_{k}=0$, and except that we allow parameters. The indices corresponding to schemata ( ), . , (IV) are those given in $[4, \S 3]$. When $s_{1}, \cdots, s_{j}, h, g_{1}^{(1)}, g_{2}^{(1)}, \cdots, g_{1}^{(j-1)}$ are respective indices of $\psi_{1}, \cdots, \psi_{j}, \chi$, $\gamma_{1}^{(1)}, \gamma_{2}^{(1)}, \ldots, \gamma_{1}^{(j-1)}$ as previously introduced, we assign $\left\langle 4+j, n+j, s_{1}, \ldots\right.$, $\left.s_{j}, h, g_{1}^{(1)}, g_{2}^{(1)}, \ldots . g_{1}^{(j-1)}\right\rangle$ as index to the function $\phi$ introduced by $\left(\mathrm{V}_{j}\right)$. In particular, this gives to $(\mathrm{V})\left(=\left(\mathrm{V}_{1}\right)\right)$ the same index as in $[4, \S 3]$.

For any fixed $l, m_{1}, \cdots, m_{l} \geqq 0$, a primitive recursive predicate $\operatorname{In}_{\mathbf{k}}^{m_{1}, \cdots, m_{l}}(b)$ may be defined to express that $b$ is an index of $\phi$ from assumed functions $\theta_{1}, \cdots, \theta_{l} k$-recursively. For each $k \geqq 1$, 


$$
\begin{aligned}
& \operatorname{In}_{k}^{m_{1}, \cdots, m_{l} l}(b) \equiv\left\{\left[\operatorname{In}^{m_{1}, \cdots, m_{l}}(b) \&(b)_{0}=0,1,2,3\right]\right. \\
& \bigvee\left[b=\coprod_{i<(b)} p_{2,1+3}^{(b)_{i}} \&(b)_{0}=4 \& \operatorname{In}_{k}^{m_{1}, \cdots, m_{l}}\left((b)_{2}\right)\right. \\
& \left.\&(i)_{2<i<(b)_{2,1}+3}\left(\operatorname{In}_{k}^{m_{1}, \cdots, m_{l}}\left((b)_{i}\right) \&(b)_{i, 1}=(b)_{1}\right)\right] \\
& \vee\left[b=\prod_{i \leq\left(j^{2}+j+4\right) / 2} p_{i}^{(b)_{i}} \&(b)_{0}=4+j\right. \\
& \&(i)_{1<i \leqq\left(j^{2}+j+4\right) / 2} \operatorname{In}_{k}^{m_{1}, \cdots, m_{l}}\left((b)_{i}\right) \\
& \&(i)_{1<i<j+2}\left((b)_{i, 1}=(b)_{1}-1\right) \&(b)_{j+2,1}=(b)_{1}+j \\
& \left.\left.\&(i)_{j+2<i \leqq\left(j^{2}+j+4\right) / 2}\left((b)_{i, 1}=(b)_{1}+1\right) \& 0<j<k+1\right]\right\},
\end{aligned}
$$

where $\operatorname{In}^{m_{1}}, \cdots, m_{l} l(b)$ is the predicate of $[4, \S 3] . \operatorname{In}_{1}^{m_{1}}, \cdots, m_{l}(b) \equiv \operatorname{In}^{m_{1}}, \cdots, m_{l}(b)$.

5.2. Let $c_{1}, c_{2}$ be indices from $\beta$ of the respective functions

$$
\alpha_{1}(x)=\beta(\text { fin }(x)), \quad\left\{\begin{array}{l}
\alpha_{2}(y, 0)=1, \\
\alpha_{2}\left(y, x^{\prime}\right)=y+o \beta(x),
\end{array}\right.
$$

where fin $(x)(\S 2)$ has the property fin $\left(n_{0}\right)=n$. Let

$$
\begin{aligned}
\eta(w, 0, n) & =n_{O}, \\
\eta\left(w, m^{\prime}, 0\right) & =0_{O}=1, \\
\eta\left(w, m^{\prime}, 1\right) & =\left\langle 0,1, \operatorname{tr}^{1}\left(c_{1}, \operatorname{sb}_{1}^{1}(w, m)\right)\right\rangle, \\
\eta\left(w, m^{\prime}, n^{\prime \prime}\right) & =\left\langle 0,1, \operatorname{tr}^{1}\left(c_{1}, \operatorname{sb}_{1}^{1}\left(\operatorname{tr}^{1}\left(c_{2}, \operatorname{sb}_{1}^{1}(w, m)\right), \eta\left(w, m^{\prime}, n^{\prime}\right)\right)\right)\right\rangle .
\end{aligned}
$$

Thus $\eta(w, m, n)$ is primitive recursive, and by the recursion theorem for primitive recursive functions there is an index $e$ of $\eta(e, m, n)$. Let $\eta(m, n)$ $=\eta(e, m, n)$.

Observe that $\eta\left(m^{\prime}, 1\right), \eta\left(m^{\prime}, n^{\prime \prime}\right)$ (shown below at the right) are each of the form $3 \cdot 5^{z}$, where $z_{x}\left(=\operatorname{pr}\left(z,\left\langle x_{0}\right\rangle\right)\right)$ for $x=0,1,2, \cdots$ are shown at the left:

$$
\begin{array}{lr}
\eta(m, 0), \eta(m, 1), \eta(m, 2), \cdots & \eta\left(m^{\prime}, 1\right), \\
1, \eta\left(m^{\prime}, n^{\prime}\right)+o \eta(m, 0), \eta\left(m^{\prime}, n^{\prime}\right)+o \eta(m, 1), \cdots & \eta\left(m^{\prime}, n^{\prime \prime}\right) .
\end{array}
$$

It follows by induction on $m$, and on $n$ for each $m$, that, for each $m, n$ : $\eta(m, n) \in O$ and $\eta(m, n)<_{o} \eta\left(m, n^{\prime}\right)$ and

$$
|\eta(m, n)|=\left\{\begin{array}{l}
n \text { if } m \cdot n=0 \\
n \cdot \omega^{m} \text { if } m, n>0
\end{array}\right.
$$

5.3. Since 1 is the first notation in the "fundamental sequence" of nota- 
tions with $\eta\left(m^{\prime}, n^{\prime \prime}\right)$ as "limit notation" (as shown above), $h_{y+o \eta\left(m^{\prime}, n\right)}(b, a)$ $=h_{y+o \eta\left(m^{\prime}, n^{\prime}\right)}(\langle b, 0\rangle, a)$. So, for $c_{3}$ an index of $\lambda b a \beta(\langle b, 0\rangle, a)$ from $\beta, h_{y+o \eta\left(m^{\prime}, n\right)}$ is primitive recursive in $h_{y+o \eta\left(m^{\prime}, n^{\prime}\right)}$ with index $c_{3}$. It follows by induction on $p$ that $h_{y+o \eta\left(m^{\prime}, n\right)}$ is primitive recursive in $h_{y+o \eta\left(m^{\prime}, n+p\right)}$ with index $v(p)$, where

$$
\begin{aligned}
v(0) & =\langle 0,2,1\rangle, \\
v\left(p^{\prime}\right) & =\operatorname{tr}^{2}\left(v(p), c_{3}\right) .
\end{aligned}
$$

Since $\eta\left(m^{\prime}, 1\right)$ is a limit notation with the fundamental sequence $\eta(m, 0)$, $\eta(m, 1), \eta(m, 2), \cdots$, we have $h_{y+o \eta(m, x)}(b, a)=h_{y+o \eta\left(m^{\prime}, 1\right)}(\langle b, x\rangle, a)$. So if $c_{4}$ is an index of $\lambda x b a \beta(\langle b, x\rangle, a)$ from $\beta$, then $\mu(x)=\operatorname{sb}_{2}^{1}\left(x, c_{4}\right)$ is an index of $h_{y+o \eta(m, x)}$ from $h_{y+o \eta\left(m^{\prime}, 1\right)}$.

If $\phi$ has $y+o \eta(m, 1)$-index $b$, it has $y+o \eta\left(m^{\prime}, 1\right)$-index $\operatorname{tr}^{2}(b, \mu(1))$. Thus $\phi$ has $y+o \eta(m+p, 1)$-index $\iota(b, p)$, where

$$
\begin{aligned}
\iota(b, 0) & =b, \\
\iota\left(b, p^{\prime}\right) & =\operatorname{tr}^{2}(\iota(b, p), \mu(1)),
\end{aligned}
$$

defines $\iota$ primitive recursively.

Homology of notation is defined for the present system $O$ in the same manner as for the former system [2, pp. 327-328]. Although the associative law " $(a+o b)+o c=a+o(b+o c)$ " does not hold in general for ordinal notations $[3$, footnote 29], it does for the corresponding $h$-types, i.e. $(a+o b)+o c$ is always homologous to $a+o(b+o c)$. The proof is by induction on $c$ over $O$. And (just as was the case for the predicates $H_{y},[2$, p. 329]) the functions $h_{y}$ depend only on the $h$-type of $y$, i.e. $h_{y}(b, a)=h_{z}(b, a)$ where $y$ and $z$ are homologous.

Now $(y+o \eta(0, n))+o \eta(0,1)=y+o \eta\left(0, n^{\prime}\right)$, and $\left(y+o \eta\left(m^{\prime}, 0\right)\right)+o \eta\left(m^{\prime}, 1\right)$ $=y+o \eta\left(m^{\prime}, 1\right)$. Using the remark on the associative law for $h$-types under to, $\left(y+o \eta\left(m^{\prime}, n^{\prime}\right)\right)+o \eta\left(m^{\prime}, 1\right)$ and $y+o \eta\left(m^{\prime}, n^{\prime \prime}\right)$ are limit notations for fundamental sequences of notations thus:

$$
\begin{array}{lr}
q_{0}, q_{1}, q_{2}, \cdots & \left(y+o \eta\left(m^{\prime}, n^{\prime}\right)\right)+o \eta\left(m^{\prime}, 1\right), \\
1, r_{0}, r_{1}, \cdots & y+o \eta\left(m^{\prime}, n^{\prime \prime}\right),
\end{array}
$$

where $q_{i}$ and $r_{i}$ are homologous, $i=0,1,2, \cdots$. Hence

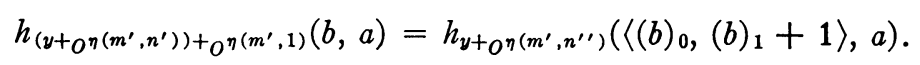

To combine the cases $m \cdot n=0$ and $m \cdot n \neq 0$, let $c_{5}$ be an index of

$$
\beta\left(\left\langle(b)_{0},(b)_{1}+1\right\rangle, a\right)
$$

from $\beta$, and put

$$
\lambda(m, n)= \begin{cases}\langle 0,2,1\rangle & \text { if } m \cdot n=0 \\ c_{5} & \text { if } m \cdot n \neq 0 .\end{cases}
$$


Then $h_{(y+o \eta(m, n))+o^{\eta}(m, 1)}$ is primitive recursive in $h_{y+o^{\eta}\left(m, n^{\prime}\right)}$ with index $\lambda(m, n)$.

Let $c_{6}=\left\langle 4,2,\langle 0,2,1\rangle,\langle 2,2,\langle 0,2,1\rangle\rangle, e_{1}\right\rangle$ be an index of $h_{y+o \eta(0, n)}$ from $h_{y+o \eta\left(0, n^{\prime}\right)}$, where $e_{1}$ is an index of $\lambda b a\langle b, a\rangle$. Using the $c_{3}$ given above, define $\rho$ primitive recursively as follows:

$$
\begin{aligned}
\rho(m, 0, b) & =b, \\
\rho\left(0, n^{\prime}, b\right) & =\operatorname{tr}^{2}\left(\rho(0, n, b), c_{6}\right), \\
\rho\left(m^{\prime}, n^{\prime}, b\right) & =\operatorname{tr}^{2}\left(b, c_{3}\right) .
\end{aligned}
$$

By induction on $m$, and on $n$ for each $m$, if a function $\alpha$ has $y$-index $b$, it has $y+o \eta(m, n)$-index $\rho(m, n, b)$.

5.4. THEOREM 3. For each $k \geqq 1$, there is a primitive recursive function $\nu_{k}(b)$ and, for each $l, m_{1}, \cdots, m_{l} \geqq 0$, a primitive recursive function $\sigma_{k}\left(b, c_{1}\right.$, $\left.\cdots, c_{l}\right)$ such that, if $\phi$ is $k$-recursive in $\theta_{1}\left(a_{1}, \cdots, a_{m_{1}}\right), \cdots, \theta_{l}\left(a_{1}, \cdots, a_{m_{l}}\right)$ with index $b$, and if, for some $y \in O, c_{1}, \cdots, c_{l}$ are $y$-indices of $\theta_{1}, \cdots, \theta_{l}$ respectively, then $\sigma_{k}\left(b, c_{1}, \cdots, c_{l}\right)$ is a $y+o \eta\left(k-2, \nu_{k}(b)\right)$-index of $\phi$.

Proof. For any $k \geqq 1$, we define $\nu_{k}$ by the course-of-values recursion

$$
\begin{aligned}
\nu_{k}(b) & =\max \left(\nu_{k}\left((b)_{2}\right), \cdots, \nu_{k}\left((b)_{\left.\left.(b)_{2,1}+2\right)\right)} \text { if }(b)_{0}=4,\right.\right. \\
& =\operatorname{sg}(k-1)+\max \left(\nu_{k}\left((b)_{2}\right), \cdots, \nu_{k}\left((b)_{\left(k^{2}+k+4\right) / 2}\right)\right) \\
& =0 \quad \text { otherwise. }
\end{aligned}
$$

The proof of the theorem and the definition of $\sigma_{k}$ now proceed by courseof-values induction on $k$.

First we treat the trivial case, $k=1$. By induction on $b, \nu_{1}(b)=0$ for all $b$, so $\eta\left(1-2, \nu_{1}(b)\right)=\eta(0,0)=1$, and $y+o \eta\left(1-2, \nu_{1}(b)\right)=y$, and it suffices to take

$$
\sigma_{1}\left(b, c_{1}, \cdots, c_{l}\right)=\operatorname{tr}^{m_{1}}, \cdots, m_{l}\left(b, c_{1}, \cdots, c_{l}\right) .
$$

For the induction step $(k>1)$, we assume the theorem to be already proved for preceding values of $k$ which are $\geqq 1$, and infer it for the given $k$ by an induction on $b$, which we present under $5+k$ cases.

CASE 0. $(b)_{0}=0$. Then $\phi=\theta_{i}, i=(b)_{2}, \nu_{k}(b)=0$. Let $\sigma_{k}\left(b, c_{1}, \cdots, c_{l}\right)=c_{i}$.

CAses $1,2,3$. $(b)_{0}=1,2,3$. Then $\phi$ is primitive recursive, $\nu_{k}(b)=0$. Let $\sigma_{k}\left(b, c_{1}, \cdots, c_{l}\right)=b$.

CASE 4. $(b)_{0}=4 . \phi\left(x_{1}, \cdots, x_{n}\right)=\psi\left(\chi_{1}\left(x_{1}, \cdots, x_{n}\right), \cdots, \chi_{m}\left(x_{1}, \cdots, x_{n}\right)\right)$, where $n=(b)_{1}, m=(b)_{2,1}$. By the hypothesis of the secondary induction on $b$, $\psi$ has $y+o \eta\left(k-2, \nu_{k}\left((b)_{2}\right)\right)$-index $\sigma_{k}\left((b)_{2}, c_{1}, \cdots, c_{l}\right)$, and $\chi_{i}$ has

$$
\begin{aligned}
y & +o \eta\left(k-2, \nu_{k}\left((b)_{2+i}\right)\right) \text {-index } \\
\sigma_{k}\left((b)_{i+2}, c_{1}, \cdots, c_{l}\right), i & =1, \cdots, m . \text { But } \\
\nu_{k}(b) & =\max \left(\nu_{k}\left((b)_{2}\right), \cdots, \nu_{k}\left((b)_{m+2}\right)\right) .
\end{aligned}
$$


So using the function $v$ defined in $5.3, \psi$ has $y+o \eta\left(k-2, \nu_{k}(b)\right)$-index $\operatorname{tr}^{2}\left(\sigma_{k}\left((b)_{2}, c_{1}, \cdots, c_{l}\right), v\left(\nu_{k}(b)-\nu_{k}\left((b)_{2}\right)\right)\right)$, and similarly with each $\chi_{i}$. Hence it suffices to take

$$
\begin{aligned}
\sigma_{k}\left(b, c_{1}, \cdots, c_{l}\right) \\
\quad=2^{4} \cdot 3^{(b)_{1}} . \prod_{1<i<(b)_{2,1+3}} p_{i} \exp \operatorname{tr}^{2}\left(\sigma_{k}\left((b)_{i}, c_{1}, \cdots, c_{l}\right), v\left(\nu_{k}(b)-\nu_{k}\left((b)_{i}\right)\right)\right) .
\end{aligned}
$$

CASE 5. $(b)_{0}=5$. Similarly we take

$$
\begin{aligned}
\sigma_{k}\left(b, c_{1}, \cdots, c_{l}\right)=2^{5} \cdot 3^{(b)_{1}} \cdot[ & {\left[5 \exp \operatorname{tr}^{2}\left(\sigma_{k}\left((b)_{2}, c_{1}, \cdots, c_{l}\right), v\left(\nu_{k}(b)-\nu_{k}\left((b)_{2}\right)\right)\right)\right] } \\
\cdot & {\left[7 \exp \operatorname{tr}^{2}\left(\sigma_{k}\left((b)_{3}, c_{1}, \cdots, c_{l}\right), v\left(\nu_{k}(b)-\nu_{k}\left((b)_{3}\right)\right)\right)\right] . }
\end{aligned}
$$

There remain $k-1$ cases for the given $k$. The treatment will be sufficiently illustrated (and the notation kept simple) by treating the two remaining cases for $k=3$ without parameters. We begin with Case 7 .

CASE 7. $(b)_{0}=7$. Then

$$
\begin{aligned}
& \phi\left(0, x_{2}, x_{3}\right)= \psi_{1}\left(x_{2}, x_{3}\right), \\
& \phi\left(x_{1}^{\prime}, 0, x_{3}\right)= \psi_{2}\left(x_{1}, x_{3}\right), \\
& \phi\left(x_{1}^{\prime}, x_{2}^{\prime}, 0\right)= \psi_{3}\left(x_{1}, x_{2}\right), \\
& \phi\left(x_{1}^{\prime}, x_{2}^{\prime}, x_{3}^{\prime}\right)= \chi\left(x_{1}, x_{2}, x_{3}, \phi\left(x_{1}, \gamma_{1}^{(1)}\left(x_{1}, x_{2}, x_{3}, \phi\left(x_{1}^{\prime}, x_{2}^{\prime}, x_{3}\right)\right),\right.\right. \\
&\left.\gamma_{2}^{(1)}\left(x_{1}, x_{2}, x_{3}, \phi\left(x_{1}^{\prime}, x_{2}^{\prime}, x_{3}\right)\right)\right), \\
& \phi\left(x_{1}^{\prime}, x_{2}, \gamma_{1}^{(2)}\left(x_{1}, x_{2}, x_{3}, \phi\left(x_{1}^{\prime}, x_{2}^{\prime}, x_{3}\right)\right)\right), \\
&\left.\phi\left(x_{1}^{\prime}, x_{2}^{\prime}, x_{3}\right)\right) .
\end{aligned}
$$

Using the hypothesis of the induction on $b$, and a construction illustrated in Case 4 , the functions $\psi_{1}, \psi_{2}, \psi_{3}, \chi, \gamma_{1}^{(1)}, \gamma_{2}^{(1)}, \gamma_{1}^{(2)}$ are primitive recursive in $h_{w}$ (where $w=y+o \eta(1, r)$ for $r=\max \left(\nu_{3}\left((b)_{2}\right), \cdots, \nu_{3}\left((b)_{8}\right)\right)$ ) with indices $s_{1}, s_{2}, s_{3}, h, g_{1}^{(1)}, g_{2}^{(1)}, g_{1}^{(2)}$, respectively, where $s_{i}=\operatorname{tr}^{2}\left(\sigma_{k}\left((b)_{i+1}, c_{1}, \cdots, c_{l}\right)\right.$, $\left.v\left(r-\nu_{3}\left((b)_{i}\right)\right)\right), i=1,2,3$, and $h, g_{1}^{(1)}, g_{2}^{(1)}, g_{1}^{(2)}$ are constructed similarly from $(b)_{5},(b)_{6},(b)_{7},(b)_{8}$.

Now observe that for each $x_{1}$ the equations of $\left(\mathrm{V}_{3}\right)$ define $\lambda x_{2} x_{3} \phi\left(x_{1}^{\prime}, x_{2} x_{3}\right)$ 2-recursively from $\psi_{2}, \psi_{3}, \chi, \gamma_{1}^{(1)}, \gamma_{2}^{(1)}, \gamma_{1}^{(2)}$, and $\lambda x_{2} x_{3} \phi\left(x_{1}, x_{2}, x_{3}\right)$. In order to exhibit the 2-recursion let

$$
\chi_{x_{1}}(a, b, c, d)=\chi\left(x_{1}, a, b, \phi\left(x_{1}, \gamma_{1}^{(1)}\left(x_{1}, a, b, d\right), \quad \gamma_{2}^{(1)}\left(x_{1}, a, b, d\right)\right), c, d\right) .
$$

For each $x_{1}, \chi_{x_{1}}$ is primitive recursive in $\chi, \lambda x_{2} x_{3} \phi\left(x_{1}, x_{2}, x_{3}\right), \gamma_{1}^{(1)}$, and $\gamma_{2}^{(1)}$, say with index $g$ (independent of $x_{1}$ ). And let

$$
\gamma_{1, x_{1}}^{(2)}(a, b, c)=\gamma_{1}^{(2)}\left(x_{1}, a, b, c\right) .
$$


Then $\lambda x_{2} x_{3} \phi\left(x_{1}^{\prime}, x_{2}, x_{3}\right)$ is introduced by the 2 -recursion

$$
\begin{aligned}
\phi\left(x_{1}^{\prime}, 0, x_{3}\right) & =\psi_{2}\left(x_{1}, x_{3}\right), \\
\phi\left(x_{1}^{\prime}, x_{2}^{\prime}, 0\right) & =\psi_{3}\left(x_{1}, x_{2}\right), \\
\phi\left(x_{1}^{\prime}, x_{2}^{\prime}, x_{3}^{\prime}\right) & =\chi_{x_{1}}\left(x_{2}, x_{3}, \phi\left(x_{1}^{\prime}, x_{2}, \gamma_{1, x_{1}}^{(2)}\left(x_{2}, x_{3}, \phi\left(x_{1}^{\prime}, x_{2}^{\prime}, x_{3}\right)\right)\right), \phi\left(x_{1}^{\prime}, x_{2}^{\prime}, x_{3}\right)\right) .
\end{aligned}
$$

And $f=\langle 6,2,\langle 0,1,1\rangle,\langle 0,1,2\rangle,\langle 0,4,3\rangle,\langle 0,3,4\rangle\rangle$ defines $\lambda x_{2} x_{3} \phi\left(x_{1}^{\prime}, x_{2}, x_{3}\right)$ from $\lambda x_{3} \psi_{2}\left(x_{1}, x_{3}\right), \lambda x_{2} \psi_{3}\left(x_{1}, x_{2}\right), \chi_{x_{1}}$ and $\gamma_{1, x_{1}}^{(2)}$ 2-recursively.

Using 5.3, $\tau_{1}\left(x_{1}\right)=\operatorname{sb}_{1}^{1}\left(\rho\left(0, x_{1}, s_{2}\right), x_{1}\right)$ is a $w+o \eta\left(0, x_{1}\right)$-index of $\lambda x_{3} \psi_{2}\left(x_{1}, x_{3}\right)$, $\tau_{2}\left(x_{1}\right)=\operatorname{sb}_{1}^{1}\left(\rho\left(0, x_{1}, s_{3}\right), x_{1}\right)$ of $\lambda x_{2} \psi_{3}\left(x_{1}, x_{2}\right), \tau_{3}\left(x_{1}\right)=\operatorname{sb}_{3}^{1}\left(\rho\left(0, x_{1}, g_{1}^{(2)}\right), x_{1}\right)$ of $\gamma_{1, x_{1}}^{(2)}, \tau_{4}\left(x_{1}\right)=\rho\left(0, x_{1}, h\right)$ of $\chi, \tau_{5}\left(x_{1}\right)=\rho\left(0, x_{1}, g_{1}^{(1)}\right)$ of $\gamma_{1}^{(1)}$, and $\tau_{6}\left(x_{1}\right)=\rho\left(0, x_{1}, g_{2}^{(1)}\right)$ of $\gamma_{2}^{(1)}$.

Now we shall work out the definition of a function $\kappa\left(x_{1}\right)$ such that, for each $x_{1}, \kappa\left(x_{1}\right)$ will be a $w+o \eta\left(0, x_{1}\right)$-index of $\lambda x_{2} x_{3} \phi\left(x_{1}, x_{2}, x_{3}\right)$. Clearly $\kappa(0)=s_{1}$. If $\kappa\left(x_{1}\right)$ is a $w+o \eta\left(0, x_{1}\right)$-index of $\lambda x_{2} x_{3} \phi\left(x_{1}, x_{2}, x_{3}\right)$, then $\tau_{7}\left(x_{1}, \kappa\left(x_{1}\right)\right)$ $=\operatorname{tr}^{6,2,4,4}\left(g, \tau_{4}\left(x_{1}\right), \kappa\left(x_{1}\right), \tau_{5}\left(x_{1}\right), \tau_{6}\left(x_{1}\right)\right)$ is such an index of $\chi_{x_{1}}$, and hence $\tau_{8}\left(x_{1}, \kappa\left(x_{1}\right)\right)=\sigma_{2}\left(f, \tau_{1}\left(x_{1}\right), \tau_{2}\left(x_{1}\right), \tau_{7}\left(x_{1}, \kappa\left(x_{1}\right)\right), \tau_{3}\left(x_{1}\right)\right)$ is a $\left(w+o \eta\left(0, x_{1}\right)\right)$ $+o \eta\left(0, \nu_{2}(f)\right)$-index of $\lambda x_{2} x_{3} \phi\left(x_{1}^{\prime}, x_{2}, x_{3}\right)$. But under the above definition of $\nu_{k}, \nu_{2}(f)=1 ; \tau_{8}\left(x_{1}, \kappa\left(x_{1}\right)\right)$ is thus a $\left(w+o \eta\left(0, x_{1}\right)\right)+o \eta(0,1)$-index of $\lambda x_{2} x_{3} \phi\left(x_{1}^{\prime}, x_{2}, x_{3}\right)$. Using the function $\lambda$ of 5.3 ,

$$
\tau_{9}\left(x_{1}, \kappa\left(x_{1}\right)\right)=\operatorname{tr}^{2}\left(\tau_{8}\left(x_{1}, \kappa\left(x_{1}\right)\right), \quad \lambda\left(0, x_{1}\right)\right)
$$

is a $w+o \eta\left(0, x_{1}^{\prime}\right)$-index of $\lambda x_{2} x_{3} \phi\left(x_{1}^{\prime}, x_{2}, x_{3}\right)$. So take $\kappa\left(x_{1}^{\prime}\right)=\tau_{9}\left(x_{1}, \kappa\left(x_{1}\right)\right)$. The functions $\tau_{1}, \cdots, \tau_{9}$ are primitive recursive and

$$
\begin{aligned}
\kappa(0) & =s_{1}, \\
\kappa\left(x_{1}^{\prime}\right) & =\tau_{9}\left(x_{1}, \kappa\left(x_{1}\right)\right),
\end{aligned}
$$

so $\kappa$ is primitive recursive.

Since $\kappa\left(x_{1}\right)$ is a $w+o \eta\left(0, x_{1}\right)$-index of $\lambda x_{2} x_{3} \phi\left(x_{1}, x_{2}, x_{3}\right), \phi\left(x_{1}, x_{2}, x_{3}\right)$ $=h_{w+o^{\eta(1,1)}}\left(\left\langle\kappa\left(x_{1}\right), x_{1}\right\rangle,\left\langle x_{2}, x_{3}\right\rangle\right)$ and (letting $q$ be an index of $\kappa$ and $e_{1}$ an index of $\lambda b a\langle b, a\rangle)$

$i=\left\langle 4,3,\langle 0,2,1\rangle,\left\langle 4,3, e_{1},\langle 4,3, q,\langle 3,3,1\rangle\rangle,\langle 3,3,1\rangle\right\rangle,\left\langle 4,3, e_{1},\langle 3,3,2\rangle,\langle 3,3,3\rangle\right\rangle\right\rangle$ is a $w+o \eta(1,1)$ index of $\phi$. But $w=y+o \eta(1, r)$. So $\operatorname{tr}^{2}(i, \lambda(1, r))$ is a $y+o \eta\left(1, r^{\prime}\right)$ index of $\phi$. Since $r^{\prime}=\nu_{3}(b), \operatorname{tr}^{2}(i, \lambda(1, r))$ is a $y+o \eta\left(1, \nu_{3}(b)\right)$-index of $\phi$. Hence it suffices to take $\sigma_{3}\left(b, c_{1}, \cdots, c_{l}\right)=\operatorname{tr}^{2}\left(i, \lambda\left(1, \nu_{3}(b)-1\right)\right)$.

For any $k>1$, the proof of Case $4+k$ proceeds similarly, and we take $\sigma_{k}\left(b, c_{1}, \cdots, c_{l}\right)=\operatorname{tr}^{2}\left(i_{k}, \lambda\left(k-2, \nu_{k}(b)-1\right)\right)$, where $i_{k}$ is a $w+o \eta(k-2,1)-$ index of $\phi\left(i_{3}=i\right)$. (If $k=2$, the proof is a bit simpler.) If parameters are allowed, slight alterations in the proof give us $i_{k}$ as a primitive recursive function of $(b)_{1}$ (the number of variables of $\phi$ ), hence as a primitive recursive function of $b$. 
The treatment of Case 6 can be described briefly in terms of the work on Case 7.

CASE 6. $(b)_{0}=6 . \phi\left(x_{1}, x_{2}\right)$ is introduced by an application of $\left(\mathrm{V}_{2}\right)$. The auxiliary functions $\psi_{1}, \psi_{2}, \chi, \gamma_{1}^{(1)}$ for $\left(\mathrm{V}_{2}\right)$ are primitive recursive in $h_{w}$ (for a $w$ defined similarly to that of Case 7). By the proof of Case 6 for $k=2$ (under the hypothesis of induction on $k) \phi$ has a $w+o \eta(0,1)$-index $i_{2}$, and hence a $w+o \eta(1,1)$-index $\iota\left(i_{2}, 1\right)$, using the $\iota$ of 5.3. As in the last step of Case 7 , we may take $\sigma_{3}\left(b, c_{1}, \cdots, c_{l}\right)=\operatorname{tr}^{2}\left(\iota\left(i_{2}, 1\right), \lambda\left(1, \nu_{3}(b)-1\right)\right)$.

For any $k>2$, the proof of Case $4+j, 1<j<k$, proceeds similarly. Here $\iota\left(i_{j}, k-j\right)$ is a $w+o \eta(k-2,1)$ index of $\phi$. Take

$$
\sigma_{k}\left(b, c_{1}, \cdots, c_{l}\right)=\operatorname{tr}^{2}\left(\iota\left(i_{j}, k-j\right), \lambda\left(k-2, \nu_{k}(b)-1\right)\right) .
$$

We exhibit the primitive recursive definition of $\sigma_{k}$ separately.

$$
\begin{aligned}
& \sigma_{k}\left(b, c_{1}, \cdots, c_{l}\right)=c_{i} \text { if }(b)_{0}=0 \&(b)_{2}=i \quad(i=1, \cdots, l), \\
& =b \text { if }(b)_{0}=1,2,3, \\
& =2^{4} \cdot 3^{(b)_{1}} \cdot \prod_{1<i<(b)_{2,1+3}} p_{i} \exp \operatorname{tr}^{2}\left(\sigma_{k}\left((b)_{i}, c_{1}, \cdots, c_{l}\right), v\left(\nu_{k}(b)-\nu_{k}\left((b)_{i}\right)\right)\right) \\
& =2^{5} \cdot 3^{(b)_{1}} \cdot\left[5 \exp \operatorname{tr}^{2}\left(\sigma_{k}\left((b)_{2}, c_{1}, \cdots, c_{l}\right), v\left(\nu_{k}(b)-\nu_{k}\left((b)_{2}\right)\right)\right)\right] \\
& \quad \cdot\left[7 \exp ^{2}\left(\sigma_{k}\right)=4,\right. \\
& \left.\left.=\operatorname{tr}^{2}\left((b)_{3}, c_{1}, \cdots, c_{l}\right), v\left(\nu_{k}(b)-\nu_{k}\left((b)_{3}\right)\right)\right)\right] \text { if }(b)_{0}=5, \\
& \left.\left.=\operatorname{tr}^{2}\left(i_{k}, \lambda\left(k \cdot-2, \nu_{k}(b)-1\right)\right)-1\right)\right) \text { if }\left(b_{0}\right)=4+j, \quad(j=2, \cdots, k-1), \\
& =0 \quad \text { otherwise. }
\end{aligned}
$$

Note that for $k=1$, this definition agrees with p. 96 (cf. $[4, \S 4]$ ).

Corollary 1. If $\phi$ is $k$-recursive, then there is an $n(n=0$, if $k=1)$ such that $\phi$ is primitive recursive in $h_{\eta(k-2, n)}$; thus for all $k>1$, the $k$-recursive functions appear in the hierarchy below the $\omega^{k-1}$ level.

Proof. If $k>1$, apply the theorem with $l=0$ and $y=0_{o}$.

6. In $[4, \S 6]$ the notion of primitive recursive degree is introduced in analogy to that of degree of recursive unsolvability (general recursive degree) of Kleene and Post [5]. Operations $\cup$ and ' are given under which formulas (1)-(12) of [5] hold.

We shall use the term degree, unless otherwise qualified, to refer to primitive recursive degree.

This system of degrees is a refinement of the system of general recursive degrees of Kleene and Post, since clearly no functions of the same degree will be of different general recursive degrees. The degrees of objects of general recursive degree $\mathbf{0}$ might be called "degrees of solvability." 
For any two functions $\alpha$ and $\beta$, either $\alpha$ is primitive recursive in $\beta$ or not, so that for each two degrees $a$ and $b$, either $a<b, a=b, a>b$, or $a \mid b$. That the fourth possibility occurs follows of course from the existence of incomparable general recursive degrees. But we shall later exhibit incomparable degrees within a single general recursive degree, i.e. incomparable degrees associated with objects having the same general recursive degree.

That there is no highest degree is an immediate consequence of the absence of a highest general recursive degree. The question whether there is a highest degree within a given general recursive degree can easily be answered in the negative. Suppose $\alpha(x)$ is of degree a within a given general recursive degree $\mathrm{d}$. Then $\operatorname{pr}^{\alpha}(b, a)$, of general recursive degree $\mathrm{d}$, is of degree $\mathbf{a}^{\prime}>\mathbf{a}$.

There are $\boldsymbol{\aleph}_{0}$ functions of each degree, and $2 \boldsymbol{\aleph}_{0}$ degrees. There are $\boldsymbol{\aleph}_{0}$ degrees within each general recursive degree since $(\leqq)$ there are only $\boldsymbol{\aleph}_{0}$ functions of each general recursive degree (by $[5,1.2]$ ) and since $(\geqq)$ there is no highest degree within any general recursive degree.

The definition of independence of degrees and the proof that for $n>2$ independence implies pairwise incomparability but not conversely translate directly from $[5,1.3]$, replacing recursive by primitive recursive. In 8.2 we will show the existence for any $n \geqq 1$, of $n$ independent degrees within any given general recursive degree, so the definition and proof apply to degrees within a given general recursive degree.

As was true for general recursive degrees, the degree of an infinite join of a set of functions is not determined by the degrees of the functions. It depends on the functions themselves and on the order in which they are joined.

7.7.1. Lemma 1. For each $l, m_{1}, \cdots, m_{l} \geqq 0$, there is a primitive recursive function $\rho(b)$ such that, if $\theta_{i}\left(x_{1}, \cdots, x_{m_{i}}\right)$ is bounded by $\beta_{i}\left(x_{1}, \cdots, x_{m_{i}}\right)$, $i=1, \cdots, l$, then $\operatorname{pr}^{\theta_{1}, \cdots, \theta_{l}}(b, a) \leqq \operatorname{pr}^{\beta_{1}, \cdots, \beta_{l}}(\rho(b), a)$ and, when $\operatorname{In}^{m_{1}}, \cdots, m l(b)$, the number $\rho(b)$ is an index from $\beta_{1}, \cdots, \beta_{l}$ of a function of $(b)_{1}$ variables which is monotone nondecreasing in each variable.

Proof. A function which fails to be monotone nondecreasing can be introduced under Schemata ( ), . . , (V) only by ( ) or (V) or by application of (IV) to previously introduced functions which fail to be monotone nondecreasing. Let $d_{i}, i=1, \cdots, l$, be an index of

$$
\lambda x_{1}, \cdots, x_{m_{i}} \sum_{y_{1} \leqq x_{1}, \cdots, y_{m_{i}} \leq x_{m_{i}}} \beta_{i}\left(y_{1}, \cdots, y_{m_{i}}\right) \text { from } \beta_{i} .
$$

Let $c$ be an index of $\lambda x y x+y$. If an index $b$ defines $\phi$ from $\theta_{1}, \cdots, \theta_{l}$ and if $(b)_{0}=5$, then Schema $(\mathrm{V})$, altered by replacing $\chi\left(x_{1}, y, x_{2}, \cdots, x_{n}\right)$ by $y+\chi\left(x_{1}, y, x_{2}, \cdots, x_{n}\right)$, introduces a function defined from $\theta_{1}, \cdots, \theta_{l}$ by

$$
\left\langle 5,(b)_{1},(b)_{2},\left\langle 4,(b)_{3,1}, c,\left\langle 3,(b)_{3,1}, 2\right\rangle,(b)_{3}\right\rangle\right\rangle .
$$

Now define by course-of-values recursion 


$$
\begin{array}{rlrl}
\rho(b) & =d_{i} \text { if }(b)_{0}=0 \&(b)_{2}=i & (i=1, \cdots, l), \\
& =b \text { if }(b)_{0}=1,2,3, & \\
& =2^{4} \cdot 3^{(b)_{1}} \cdot \prod_{1<i<(b)_{2,1+3}} p_{i}^{\rho\left((b)_{i}\right)} & \text { if }(b)_{0}=4, \\
& =\left\langle 5,(b)_{1}, \rho\left((b)_{2}\right),\left\langle 4,(b)_{3,1}, c,\left\langle 3,(b)_{3,1}, 2\right\rangle, \rho\left((b)_{3}\right)\right\rangle\right\rangle & \text { if }(b)_{0}=5, \\
& =0 \quad \text { otherwise. }
\end{array}
$$

The function $\rho$ is primitive recursive. An induction on $b$ shows that, if $\operatorname{In}^{m_{1}}, \cdots, m l(b)$, the function defined by $\rho(b)$ from $\beta_{1}, \cdots, \beta_{l}$ is monotone nondecreasing and simultaneously (using the monotonicity) that it bounds the function defined by $b$ from $\theta_{1}, \cdots, \theta_{l}$. If $\overline{\operatorname{In}}^{m_{1}}, \cdots, m l(b)$, then $\operatorname{pr}^{\theta_{1}, \cdots, \theta_{l}}(b, a)=0$.

7.2. To any function, predicate or set there is an object of each of the other two kinds having the same general recursive degree $[5,1.2]$. This is not the case for primitive recursive degrees.

THEOREM 4. If $\alpha(x)$, of degree $\mathbf{a}$, is a function which majorizes the one-place primitive recursive functions, then there is no predicate or set of degree a.

Proof. The degree of a predicate or set is the degree of its representing function $\theta$. To apply Lemma 1 (7.1) with $l=1$, let $\beta(x)=1$ since for all $x, \theta(x) \leqq 1$. Then to each function $\phi(x)$ primitive recursive in a predicate or set (i.e. primitive recursive, say with index $e$, in $\theta$ ) there is a primitive recursive function $\operatorname{pr}^{\beta}(\rho(e),\langle x\rangle)$ which bounds $\phi(x)$. Thus a function $\alpha(x)$ which majorizes all the one-place primitive recursive functions cannot be primitive recursive in any such $\theta$ (hence not in any predicate or set) and so cannot be of the same degree. This completes the proof.

Observe that if $d>a, d$ is also the degree of a function which majorizes the one-place primitive recursive functions since, if $\delta$ is of degree $\mathbf{d}$, then $\langle\alpha(x), \delta(x)\rangle$ is of degree $\mathrm{d}[5,(7)]$ and for all $x,\langle\alpha(x), \delta(x)\rangle \geqq \alpha(x)$.

It can be noted, in addition, that $a$ is actually incomparable with the degree of some predicates. For example, define $v(x)=\overrightarrow{\mathrm{s}} g\left(\operatorname{pr}^{\alpha}(x,\langle x\rangle)\right)$; then $v(x)$ takes on only values 0 and 1 (and is thus the representing function of a predicate $U(x)$ ), and differs from every function primitive recursive in $\alpha$. The degree of $U$ is incomparable with that of $\alpha$. And if $\alpha$ is recursive (e.g. $\alpha(x)$ $=\sum_{a, b \leq x} h_{2}(b, a)$, which is of degree $\left.0^{\prime}\right)$ then $U$ is recursive. The degrees of $\alpha$ and of $U$ may thus both be within general recursive degree 0 .

8. 8.1. For the purposes of Theorem 5 we show that for a given function $\alpha(x)$ a function $\pi(b, a)$, primitive recursive in $\operatorname{pr}^{\alpha}(b, a)$, can be defined which, when $\operatorname{In}^{1}(b)$, gives for all functions $\theta(x)$ which take on only values 0 and 1 , an upper bound for the values of $x$ for which values of $\langle\alpha(x), \theta(x)\rangle$ are required for the computation (based on index $b$ ) of the function value $\operatorname{pr}^{\lambda x\langle\alpha(x), \theta(x)\rangle}(b, a)$. In other words, if

$$
\begin{aligned}
(x)_{x<\pi(b, a)}\left[\phi_{1}(x)\right. & =\phi_{2}(x) \&\left(\phi_{i}(x)\right)_{0}=\alpha(x) \&\left(\phi_{i}(x)\right)_{1} \leqq 1 \& \phi_{i}(x) \\
& \left.=\left\langle\left(\phi_{i}(x)\right)_{0},\left(\phi_{i}(x)\right)_{1}\right\rangle\right], \quad i=1,2,
\end{aligned}
$$

then $\operatorname{pr}^{\phi_{1}}(b, a)=\operatorname{pr}^{\phi_{2}}(b, a)$. 
To use Lemma 1 (7.1) with $l=m_{1}=1$, choose $\beta(x)=\langle\alpha(x), 1\rangle$. Thus, for every function $\theta(x)$ which takes on only values 0 and 1 , for all $x, \beta(x)$ $\geqq\langle\alpha(x), \theta(x)\rangle$, and $\psi(b, a)=\operatorname{pr}^{\beta}(\rho(b), a) \geqq \operatorname{pr}^{\lambda x\langle\alpha(x), \theta(x)\rangle}(b, a)$.

Then define $\pi$ as follows:

$$
\begin{aligned}
& \pi(b, a)= a \text { if }(b)_{0}=0, \\
&= \max _{i, j}\left[\pi\left((b)_{2}, i\right), \pi\left((b)_{j}, a\right)\right] \text { where } i \leqq \prod_{k<(b)_{2,1}} p_{k}^{\psi\left((b)_{k+3}, a\right)} \\
& \text { and } 2<j<(b)_{2,1}+3 \text { if }(b)_{2,1}>0 \text { and }(b)_{0}=4, \\
&=\pi\left((b)_{2}, \prod_{k<(b)_{1} \dot{-1}} p_{k}^{(a)_{k+1}}\right) \text { if }(b)_{0}=5 \&(b)_{1}>1 \&(a)_{0}=0, \\
&=\max _{j}\left[\pi(b,[a / 2]), \pi\left((b)_{3}, 2^{(a)_{0} \dot{-1}} \cdot 3^{j} \cdot \prod_{1<k \leqq(b)_{1}} p_{k}^{(a)_{k} \dot{1}}\right)\right] \\
& \quad \text { where } j \leqq \psi(b,[a / 2]) \text { if }(b)_{0}=5 \&(a)_{0}>0 \\
&\left.=0 \text { otherwise (including when }(b)_{0}=1,2,3\right) .
\end{aligned}
$$

A course-of-values induction on index $b$ shows that $\pi(b, a)$ has the required property. If $(b)_{0}=0,1,2,3$, the property clearly is obtained. If $(b)_{0}=4$ we need only observe that it suffices to choose $\pi(b, a)$ no smaller than

$\max \left[\pi\left((b)_{2}, \prod_{k<m} p_{k} \exp \operatorname{pr}^{\lambda x\langle\alpha(x), \theta(x)\rangle}\left((b)_{k+3}, a\right)\right), \pi\left((b)_{3}, a\right), \cdots, \pi\left((b)_{m+2}, a\right)\right]$,

where $m=(b)_{2,1}$. Since $\psi(b, a) \geqq \operatorname{pr}^{\lambda x\langle\alpha(x), \theta(x)\rangle}(b, a)$, the number

$$
\prod_{k<m} p_{k} \exp \operatorname{pr}^{\lambda x\langle\alpha(x), \theta(x\rangle)\left((b)_{k+3}, a\right)}
$$

lies among the values of $i$ used under the fourth case in the definition of $\pi(b, a)$. Similarly if $(b)_{0}=5$.

Now $\beta(x)=\langle\alpha(x), 1\rangle$ is primitive recursive in $\alpha(x)$. So by $(10)$ of $[4, \S 6]$, $\operatorname{pr}^{\beta}(b, a)$ is primitive recursive in $\operatorname{pr}^{\alpha}(b, a)$. Hence $\psi(b, a)$ is primitive recursive in $\operatorname{pr}^{\alpha}(b, a)$. Observe that the definition of $\pi(b, a)$ from $\psi(b, a)$, and thence from $\operatorname{pr}^{\alpha}(b, a)$ is an unnested course-of-values double recursion: for given $b$ and $a$, the value $\pi(b, a)$ is obtained primitive recursively from values $\pi(b, y)$, where $y<a$, and values $\pi(x, z)$, where $x<b$ and $z$ is given primitive recursively from $\operatorname{pr}^{\alpha}(b, a)$. Hence by combining the methods of $[6, \S \S 3$ and 6], $\pi(b, a)$ is primitive recursive in $\operatorname{pr}^{\alpha}(b, a)$.

8.2. THEOREM 5. For any $n \geqq 1$, given any function $\alpha(x)$, of degree $\mathbf{a}$, which majorizes the primitive recursive functions (e.g. $\left.\mathbf{a}=0^{\prime}\right)$, there exist functions $\theta_{1}(x), \cdots, \theta_{n}(x)$ such that:

(1) for $i=1, \cdots, n, \theta_{i}$ is primitive recursive in $\operatorname{pr}^{\alpha}(b, a)$,

(2) for $i=1, \cdots, n, \theta_{i}$ is not primitive recursive in $\alpha, \theta_{1}, \cdots, \theta_{i-1}$, $\theta_{i+1}, \cdots, \theta_{n}$, and 
(3) $\alpha$ is not primitive recursive in $\theta_{1}, \cdots, \theta_{n}$.

Proof. For simplicity, we illustrate the proof with $n=2$. For larger $n$, replace $\langle\alpha(x), \theta(x)\rangle$ in the definition of $\pi(b, a)$ by

$$
\left\langle\alpha(x), \theta_{1}(x), \theta_{2}(x), \cdots, \theta_{n-1}(x)\right\rangle
$$

and let the $\beta(x)$ for the application of Lemma 1 (7.1) instead be $2^{\alpha(x)} \cdot \prod_{0<i<n} p_{1}^{1}$.

Let $\pi(b, a)$ be defined as in 8.1 for the $\alpha(x)$ of our theorem. Let

$$
\begin{aligned}
\nu(0) & =0, \\
\nu\left(g^{\prime}\right) & =\tilde{\pi}\left([g / 2]^{\prime}, \nu(g)^{\prime}\right)+g^{\prime} .
\end{aligned}
$$

The function $\tilde{\pi}(b, a)[1$, p. 291] is primitive recursive in $\pi(b, a)$ and is monotone nondecreasing in both variables. Thus $\nu(g)$ is primitive recursive in $\pi(b, a)$, hence in $\operatorname{pr}^{\alpha}(b, a)$, and is monotone increasing. Furthermore, for all $b, a^{\sim}\left(b^{\prime}, a^{\prime}\right)>\pi(b, a)$; so for any $g$ and for any $\theta$ which takes on only values 0 and 1 , the computation based on index $b$ of $\operatorname{pr}^{\lambda x\langle\alpha(x), \theta(x)\rangle}(b, \nu(g)), b=[g / 2]$, requires only values of $\alpha(x)$ and of $\theta(x)$ for $x<\nu\left(g^{\prime}\right)$.

Suppose a function $\phi(x)$ is primitive recursive in $\langle\alpha(x), \theta(x)\rangle$ with index $b$ and that $\theta(x)$ is a primitive recursive function with index $t$. Then $\phi$ is primitive recursive in $\alpha$ with index $\operatorname{tr}^{1}\left(b,\left\langle 4,1, e_{1},\langle 0,1,1\rangle, t\right\rangle\right)$, where $e_{1}$ is an index of $\lambda y z\langle y, z\rangle$. In particular, if $\theta(x)=\lambda x(c)_{x}-1$, then $t=\operatorname{sb}_{1}^{1}(e, c)$ for $e$ an index of $\lambda c x(c)_{x}-1$ (cf. $\left.[4, \S 10]\right)$. So letting

$$
\epsilon(b, c)=\operatorname{tr}^{1}\left(b,\left\langle 4,1, e_{1},\langle 0,1,1\rangle, \operatorname{sb}_{1}^{1}(e, c)\right\rangle\right),
$$

$\epsilon$ is primitive recursive and

$$
\operatorname{pr}^{\lambda x\left\langle\alpha(x),(c)_{z} \dot{-1}\right\rangle}(b, a)=\operatorname{pr}^{\alpha}(\epsilon(b, c), a) .
$$

We now proceed to define the functions $\theta_{1}$ and $\theta_{2}$. First let

where

$$
\begin{gathered}
\zeta(0)=2 \exp \prod_{i<\nu(1)} p_{i}, \\
\zeta\left(g^{\prime}\right)= \begin{cases}\zeta(g) \cdot p_{0^{\prime}}^{\gamma_{1}(o)} & \text { if } g=2 b+1, \\
\zeta(g) \cdot p_{0^{\prime}}^{\gamma_{2}(o)} & \text { if } g=2 b,\end{cases}
\end{gathered}
$$

and

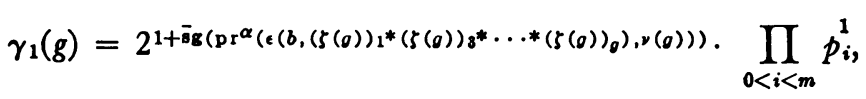

$$
\gamma_{2}(g)=2^{1+\overline{\mathrm{B}}_{\mathrm{g}}\left(\mathrm{pr}^{\alpha}\left(\epsilon\left(b,(\zeta(o))_{0}^{*}(\zeta(o)) 2^{*} \cdots *(\zeta(o))_{g}\right), \nu(o)\right)\right)} \cdot \prod_{0<i<m} p_{i}^{1},
$$

and $m=\nu(g+2)-\nu(g)$. Observe that, for $h \geqq i,(\zeta(h))_{i}=(\zeta(i))_{i}$; and furthermore that, for $g$ odd $[g$ even],

$$
(\zeta(g))_{1} *(\zeta(g))_{3} * \cdots *(\zeta(g))_{0}\left[(\zeta(g))_{0} *(\zeta(g))_{2} * \cdots *(\zeta(g))_{0}\right]
$$


is a product of the first $\nu\left(g^{\prime}\right)$ primes to nonvanishing exponents, where for even $y<g$ [odd $y<g$ ] the exponent of $p_{\nu(y)}$ is

$$
\begin{gathered}
1+\overline{\operatorname{s}} g\left(\operatorname{pr}^{\alpha}\left(\epsilon\left([y / 2],(\zeta(y))_{0} *(\zeta(y))_{2} * \cdots *(\zeta(y))_{y}\right), \nu(y)\right)\right) \\
{\left[1+\overline{\operatorname{s}} g\left(\operatorname{pr}^{\alpha}\left(\epsilon\left([y / 2],(\zeta(y))_{1} *(\zeta(y))_{3} * \cdots *(\zeta(y))_{y}\right), \nu(y)\right)\right)\right], \text { all other }}
\end{gathered}
$$

exponents being 1. The function $\zeta$ is primitive recursive in $\operatorname{pr}^{\alpha}$, since the expression $(\zeta(g))_{1} *(\zeta(g))_{3} * \cdots *(\zeta(g))_{\theta}$ in $\gamma_{1}(g)$ can be written

$$
\prod_{i \leq[\sigma / 2]}^{*}(\zeta(g))_{2 i+1}
$$

where $\Pi^{*}$ is defined from $*$ in the same way as $\Pi$ from $\cdot[1$, p. 224], and similarly in $\gamma_{2}(g)$.

Now let

$$
\begin{aligned}
& \theta_{1}(x)=\left((\zeta(2 x+1))_{1} *(\zeta(2 x+1))_{3} * \cdots *(\zeta(2 x+1))_{2 x+1}\right)_{x}-1, \\
& \theta_{2}(x)=\left((\zeta(2 x))_{0} *(\zeta(2 x))_{2} * \cdots *(\zeta(2 x))_{2 x}\right)_{x}-1 .
\end{aligned}
$$

Since $\theta_{1}$ and $\theta_{2}$ are primitive recursive in $\zeta$, they are primitive recursive in $\operatorname{pr}^{\alpha}(b, a)$. Also $\theta_{1}(x)$ and $\theta_{2}(x)$ take on only values 0 and 1 . Furthermore, if $g=2 b+1$, then, using the above observations and the monotonicity of $\nu(g)$, and writing $\bar{\theta}_{1}(x)=\prod_{i<x} p_{i}^{\theta_{1}(i)+1}$ (cf. [2, footnote 2]),

$$
\begin{aligned}
\theta_{2}(\nu(g)) & =\overline{\operatorname{s}} g\left(\operatorname{pr}^{\alpha}\left(\epsilon\left(b,(\zeta(g))_{1} *(\zeta(g))_{3} * \cdots *(\zeta(g))_{g}\right), \nu(g)\right)\right) \\
& =\bar{s} g\left(\operatorname{pr}^{\alpha}\left(\epsilon\left(b, \bar{\theta}_{1}\left(\nu\left(g^{\prime}\right)\right)\right), \nu(g)\right)\right) \\
& \left.=\overline{\operatorname{s}} g\left(\operatorname{pr}^{\lambda x\left\langle\alpha(x),\left(\bar{\theta}_{1}\left(\nu\left(g^{\prime}\right)\right)\right)_{x}-1\right\rangle}\right\rangle(b, \nu(g))\right) .
\end{aligned}
$$

Now by a property of $\nu(g)$, since $\theta_{1}(x)$ takes on only values 0 and 1 , the computation based on index $b=[g / 2]$ of $\operatorname{pr}^{\lambda x\left\langle\alpha(x), \theta_{1}(x)\right\rangle}(b, \nu(g))$ requires only values of $\alpha(x)$ and of $\theta_{1}(x)$ for $x<\nu\left(g^{\prime}\right)$. Hence $\theta_{2}(\nu(g))=\bar{s} g\left(\operatorname{pr}^{\lambda x\left\langle\alpha(x), \theta_{1}(x)\right\rangle}(b, \nu(g))\right)$. Thus $\theta_{2}(x)$ differs from every one-place function primitive recursive in $\left\langle\alpha(x), \theta_{1}(x)\right\rangle$ for at least one value of $x$ and so is not primitive recursive in $\left\langle\alpha(x), \theta_{1}(x)\right\rangle$. Similarly $\theta_{1}(x)$ is not primitive recursive in $\left\langle\alpha(x), \theta_{2}(x)\right\rangle$. Thus conditions (1) and (2) are satisfied.

To show that condition (3) is satisfied we apply Lemma $1(7.1)$ as in the proof of Theorem 4. Recall that for all $x, \theta_{1}(x)<2$ and $\theta_{2}(x)<2$. Since the primitive recursive (constant) function $\langle 1,1\rangle$ bounds $\left\langle\theta_{1}(x), \theta_{2}(x)\right\rangle$, each function primitive recursive in $\left\langle\theta_{1}(x), \theta_{2}(x)\right\rangle$ is bounded by a primitive recursive function by Lemma 1 . By hypothesis, $\alpha(x)$ majorizes the one-place primitive recursive functions, and hence $\alpha(x)$ is not primitive recursive in $\left\langle\theta_{1}(x), \theta_{2}(x)\right\rangle$. This completes the proof of the theorem.

The arguments which yield Corollaries 1-3, [5, 2.2], may now be used to give three analogous corollaries in which degree is understood to mean primitive recursive degree and the degree a satisfies the condition of the theorem. 
No analog of Post's problem [5, p. 391] arises for this system of degrees.

Theorem 5 and its proof may be altered slightly to eliminate the function $\alpha$ and to produce, for any $n \geqq 1$, functions $\theta_{i}$ with independent degrees $t_{i}$, where $\mathbf{t}_{i}<0^{\prime}, i=1, \cdots, n$.

Theorem 5 (CONCLUded). For any $n \geqq 1$, there exist functions $\theta_{1}(x), \cdots$, $\theta_{n}(x)$ such that: (1) for $i=1, \cdots, n, \theta_{i}$ is primitive recursive in $\mathrm{pr}(b, a),(2)$ for $i=1, \cdots, n, \theta_{i}$ is not primitive recursive in $\theta_{1}, \cdots, \theta_{i-1}, \theta_{i+1}, \cdots, \theta_{n}$.

Proof. The proof is essentially that just given with the last paragraph eliminated and the following additional alterations. Let $\alpha(x)=1$. In redefining $\pi(b, a)$ choose $\beta(x)=\langle 1,1\rangle$. Then $\pi(b, a)$ is primitive recursive in $\operatorname{pr}(b, a)$. The primitive recursive function $\epsilon(b, c)$ is redefined replacing

$$
\left\langle 4,1, e_{1},\langle 0,1,1\rangle, t\right\rangle \text { by }\left\langle 4,1, e_{1},\langle 2,1,1\rangle, t\right\rangle \text {. }
$$

Finally, replace $\operatorname{pr}^{\alpha}$ in the exponent of 2 in both $\gamma_{1}(g)$ and $\gamma_{2}(g)$ by pr.

Corollaries 2 and 3 may then be amended to allow a to be any degree.

8.3. The upper semi-lattice of general recursive degrees is shown not to be a lattice, according to [5, Theorem 3$]$. The proof establishes that the upper semi-lattice of (primitive recursive) degrees is also not a lattice (leaving the question unanswered concerning degrees within a given general recursive degree). For the recursiveness in $B_{1}$ and $B_{2}$ mentioned in (B) and (C) of $[5$, Theorem 3$]$ is actually shown to be primitive $[5$, p. 405 and p. 406 lines 14-15], since, for $j \leqq k, L_{j}$ is primitive recursive in $L_{k}$ (using [5, p. 384] lines $1-3 k-j$ times).

\section{BiBLIOGRAPHY}

1. S. C. Kleene, Introduction to metamathematics, New York and Toronto, Van Nostrand, Amsterdam, North Holland Publishing Co. and Groningen, Noordhoff, 1952, 10+550 pp.

2. - Arithmetical predicates and function quantifiers, Trans. Amer. Math. Soc. vol. 79 (1955) pp. 312-340.

3. - On the forms of the predicates in the theory of constructive ordinals (second paper), Amer. J. Math. vol. 77 (1955) pp. 405-428.

4. - Extension of an effectively generated class of functions by enumeration, forthcoming in Coll. Math. (Wroclaw) vol. 6.

5. S. C. Kleene and Emil L. Post, The upper semi-lattice of degrees of recursive unsolvability, Ann. of Math. vol. 59 (1954) pp. 379-407.

6. R. Péter, Rekursive Funktionen, Budapest, Akademischer Verlag, 1951, 206 pp.

Michigan State University,

EAst LANSING, Michigan

UNIVERSITY OF WISCONSIN,

MADISON, Wisconsin 